\title{
ETIOLOGICAL STRUCTURE OF INTESTINAL INFECTIONS IN THE "PROF. DR. STOYAN KIRKOVICH "AD (UNIVERSITY) - STARA ZAGORA HOSPITAL IN 2013
}

\author{
R. Yordanova*, I. Lyutskanova, P. Georgieva, M. Boycheva, S. Isencik \\ Department of Microbiology, Faculty of Medicine, Trakia University, Stara Zagora, Bulgaria
}

\begin{abstract}
Isolates from fecal samples of patients with diarrhea syndrome from "Prof. Dr. Stoyan Kirkovich" AD (University) - Strara Zagora Hospital were included in our study for 2013. We examinated 2292 fecal samples and identified 93 strains. The most common strains were from genus Shigella and genus Salmonella, followed by diarrhea-causing E. coli, Y. enterocolitica and P. aeruginosa. Each of the isolates had been tested to antimicrobials.
\end{abstract}

Key words: Shigella spp., Salmonella spp., E. coli, Y. enterocolitica, P. aeruginosa

\section{INTRODUCTION}

Infectious diseases with leading diarrhea syndrome are global health problem (1).

Many microorganisms can cause infections of the gastrointestinal tract- food-born and toxicoinfections that proceed clinically as gastroenteritis and enterocolitis- Salmonella spp., Shigella spp., E.coli, S.aureus, B.cereus, C.botulinum, and less $Y$. enterocolitica, $C$. difficile, $\quad$ Campylobacter spp., V.parahaemolyticus, A.hydrophila, P.shigeloides, etc. $(2,3)$.

The development and the severity of disease depends on the causative agents, as well as susceptibility of the host, personal and communal hygiene, age, state of the immunity etc. (4) The occurrence of the disease depends on the infectious dose (5).

\footnotetext{
Aim

The aim of this study is to determinate the etiological structure and the antibiotic susceptibility of bacterial intestinal infections in "Prof. Dr. Stoyan Kirkovich" AD (University) - Strara Zagora Hospital in 2013.

*Correspondence to: : Rozalina Yordanova, Bulgaria, , Trakia University, Faculty of MedicineStara Zagora, Department of Microbiology, 11

“Armejska” Str., 6000 Stara Zagora, BULGARIA,

E-mail: rozalina_@abv.bg, mobil:0883335099
}

\section{MATERIALS AND METHODS}

During the analyzed period we have studied 2292 fecal samples of patients admitted in Clinic of Infectious diseases, Neonatology Clinic, Gastroenterology, and Dermatology Clinic. There were 93 isolates identified. We used conventional methods for the diagnosticscultural methods biochemical methods and serotyping. The cultivation was done on Blood agar, Mac Conkey agar, Deoxycholatecitrate agar, Eosinmethyleneblue agar (Levine), Selenite Broth. Were used also manual and semi-automated and systems (Crystal GN, mini API-ID 32 E, rapid ID 32E) (6). The antibiotic susceptibility was determined on MullerHinton agar by DDM (Disk Diffusion Method) and DDMS (Double Disk Method of Synergism) (7).

\section{RESULTS AND DISCUSSION}

Etiological structure of clinically important pathogens with diarrhea syndrome is presented in Figure 1.

The specimen distribution of isolated Shigella strains is presented in Figure 2. The distribution of genus Shigella is as follows: $(S$. flexneri- 46 isolates (81\%), S. dysenteriae3- 6 isolates (10\%), S. sonnei- 4 isolates (7\%), S. dysenteriae 4- 1 isolate (2\%). 


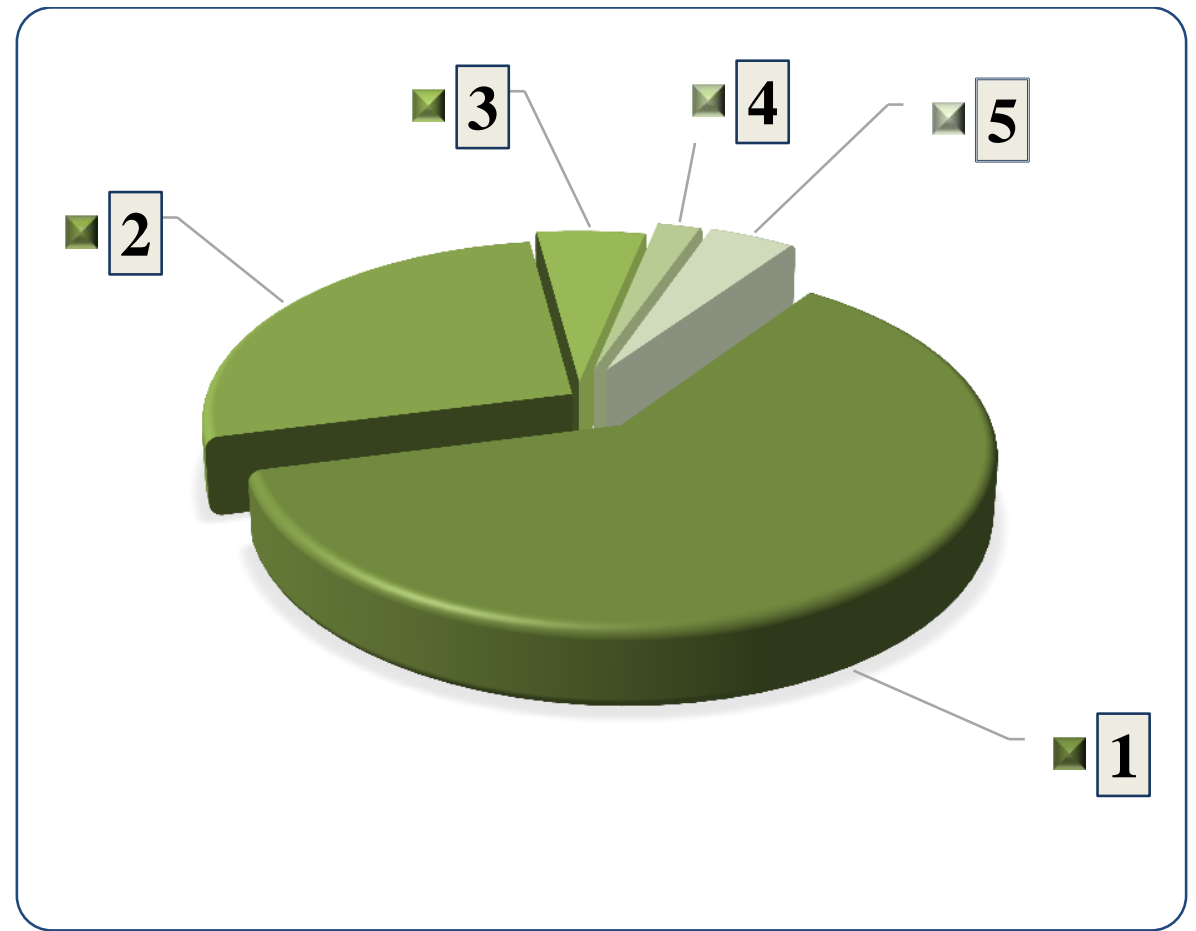

Figure 1. Etiological structure of intestinal infections in the "Prof. Dr. Stoyan Kirkovich "AD (University) Stara Zagora Hospital in 2013. $\mathrm{n}=93$
1. Shigella $61 \%$
2. Salmonella $27 \%$
3. E.coli $6 \%$
4. Y. enterocolitica $2 \%$
5. P. aeruginosa $4 \%$

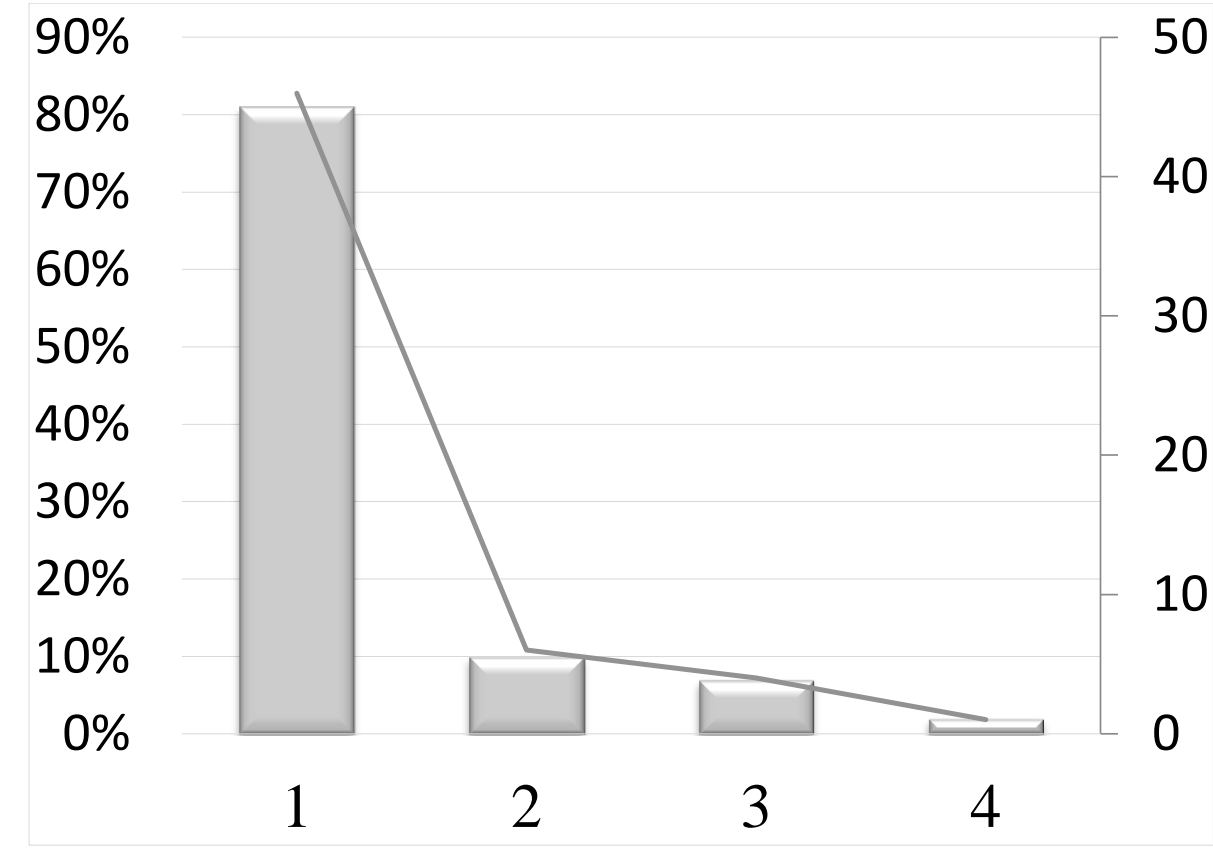

Figure 2. Distribution of isolated Shigella species.

$\begin{array}{llrr}\text { 1. } & \text { S. flexneri } & 81 \% & 46 \\ \text { 2. } & \text { S.dysenteriae3 } & 10 \% & 6 \\ \text { 3. } & \text { S. sonnei } & 7 \% & 4 \\ \text { 4. } & \text { S.dysenteriae4 } & 2 \% & 1\end{array}$

The predominant Salmonella serovars are presented in Figure 3. Among the genus Salmonella the prevalence serotype is
S. Typhimurium- 14 isolates (56\%), followed by $S$. Enteritidis- 8 isolates (32\%), S.4, 5, 12:i 2 isolates (8\%), S. Indiana- 1 isolate (4\%). 


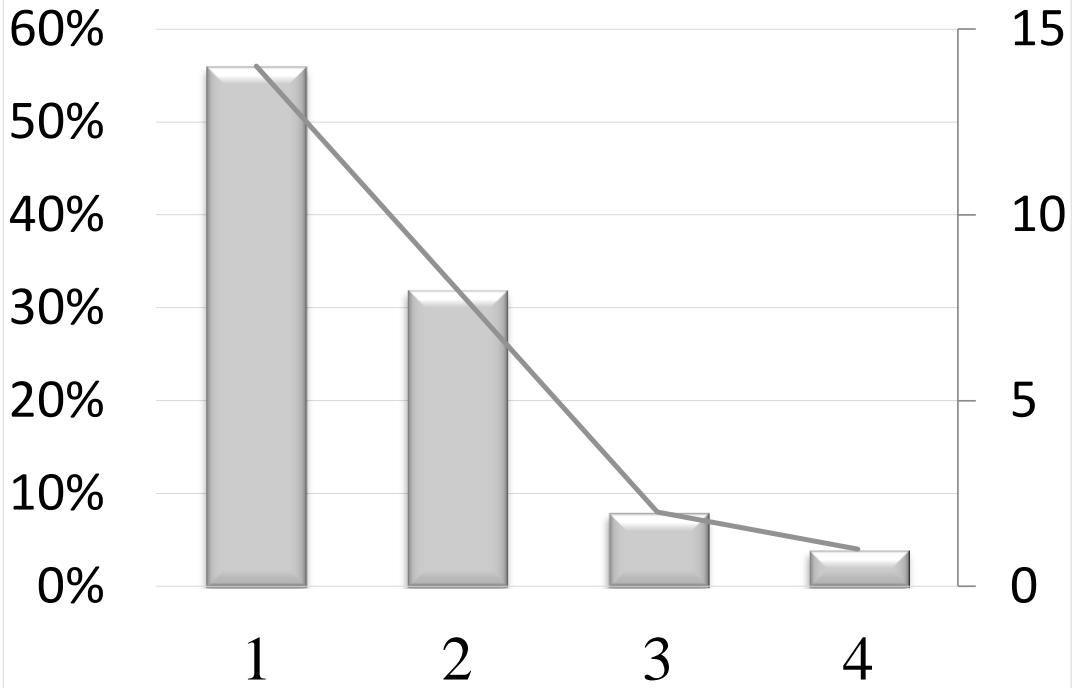

Figure 3. Distribution of isolated Salmonella serotypes.

$\mathrm{n}=25$

1. S. Typhimurium $56 \% \quad 14$

2. S. Enteritidis $32 \% \quad 8$

3. S. $4,5,12: \mathrm{I} \quad 8 \% \quad 2$

4. S. Indiana $4 \% \quad 1$

Among diarrhea-causing E. coli (5 isolates) the distribution is as follows: ETEC $O 6 \mathrm{~K}$ - 2 isolates, ETEC $O 78 \mathrm{~K} 80$ - 1 isolate, EHEC- $O$ 157 H 7 - 1 isolate.

We identified two cases with diarrhea syndrome, caused by $Y$. enterocolitica, without epidemiological connection between them.

$P$. aeruginosa were identified in four cases with diarrhea.

The case of one-year child (K. S. D.) was of a particular interest. The child was admitted in CIB II (Clinic of Infectious diseases) for Rotavirus enterocolitis. After ten days of hospitalization at the clinic we isolated $S$. flexneri from fecal samples. The child had treated etiologically and then discharged from the clinic. When the control cultures were made additionally $S$ Typhimurium was isolated. Both strains S.flexneri and S. Typhimurium with their antimicrobial susceptibility patterns were previously isolated from two more children. Perhaps, it is a case of nosocomial infection with S. Typhimurium and S.flexneri. The strains were sent and confirmed by the Reference Laboratory of NCIPD (National Center of Infectious and Parasitic Diseases).

The analyses of antimicrobial resistance of the isolated strains showed single cases of ESBLs positive Enterobacteriaceae: $S$. sonnei- 1 isolate, Salmonella 4, 5, 12:i- 1 isolate, EPEC $086 \mathrm{~K} 61$ - 1 isolate.

\section{CONCLUSION}

During the studied period the leading causative agents of bacterial intestinal infections (enterocolitis and gastroenteritis), incl. nosocomial infection are: $S$. flexneri and $S$. Typhimurium. Strains were isolated all year long and the peak is June-October.

\section{REFERENCES}

1. Patricia Tille, Bailey \& Scott's Diagnostic Microbiology, $13^{\text {th }}$ Edition, pp 945, 2014

2. Prof. G. Mitov, Canev, Docheva, Avramova, Mitov, Microbiology, ARSO Sofia, pp 631, 2000

3. Connie R. Mahon, Donald C. Lehman, George Manuselis, Texbook of Diagnostic Microbiology, $5^{\text {th }}$ Edition, pp 835, 2015

4. G. Lazarova, K. Rachkova, D. Rukanova, I. Dukova- Etiological structure of diarrheal syndrome in patients hospitalized at the Infectious disease clinic in Regional Hospital- Stara Zagora- 1976-1986Epidemiology, Infectious Diseases and Microbiology, 1987, № 4, 32-37 [4]

5. Robert W. Bauman, Microbiology with Diseases by Taxonomy, $4^{\text {th }}$ Edition, pp 444 , 2014

6. Prof. St. Petrovski, MD, Practical Clinical Microbiology Znanie Ltd., pp 49, 1999

7. The Kauffman and White classification scheme for summarizes antigenic formulae of all Salmonella serovars- $9^{\text {th }}$ edition, 2007

8. Lippincott Williams and Wilkins; Washington Winn, JR., Stephen Allen, William Janda, Elmer Koneman, Gary Procop, Paul Schreckenberger, Gail Woods, Koneman's Color Atlas and Textbook of Diagnostic Microbiology, sixth edition, pp 983, 2005 
YORDANOVA R., et al. 